\title{
Multiscale Discretization Scheme Based on the Rayleigh Quotient Iterative Method for the Steklov Eigenvalue Problem
}

\author{
Hai Bi and Yidu Yang \\ School of Mathematics and Computer Science, Guizhou Normal University, Guiyang 550001, China
}

Correspondence should be addressed to Hai Bi, junglebee@163.com

Received 27 April 2012; Accepted 9 July 2012

Academic Editor: J. Jiang

Copyright (c) 2012 H. Bi and Y. Yang. This is an open access article distributed under the Creative Commons Attribution License, which permits unrestricted use, distribution, and reproduction in any medium, provided the original work is properly cited.

This paper discusses efficient numerical methods for the Steklov eigenvalue problem and establishes a new multiscale discretization scheme and an adaptive algorithm based on the Rayleigh quotient iterative method. The efficiency of these schemes is analyzed theoretically, and the constants appeared in the error estimates are also analyzed elaborately. Finally, numerical experiments are provided to support the theory.

\section{Introduction}

Steklov eigenvalue problems have several deep applications both in physical and mechanical fields. For instance, they are found in the study of surface waves (see [1]), in the analysis of stability of mechanical oscillators immersed in a viscous fluid (see [2]), and in the study of the vibration modes of a structure in contact with an incompressible fluid (see, e.g. [3]). Thus, numerical methods for Steklov eigenvalue problems have attracted more and more scholars' attention in recent years, for example, see [4-12].

However, in practical applications, it is a challenging task to adopt efficient numerical methods to reduce the computational costs, such as the CPU time and the storage requirement, without any loss of the accuracy of numerical approximation. A two-grid finite element discretization scheme is considered one of these efficient methods. This discretization technique was first introduced by $\mathrm{Xu}[13,14]$ for nonsymmetric and nonlinear elliptic problems, and due to its outstanding performance in computation, it has been successfully applied and further investigated for many other problems, for example, Poisson eigenvalue equations and integral equations in [15], nonlinear eigenvalue problems in [16], Schrödinger equation in $[17,18]$, Stokes equations in [19], and so forth. As for the Steklov eigenvalue 
problem, Li and Yang [20], Bi and Yang [21] established conforming and nonconforming finite element two-grid discretization scheme, respectively, and obtained satisfactory results. So far, to the best of our knowledge, there have been developed two kinds of two-grid discretization scheme for eigenvalue problems. The first type is contributed by $\mathrm{Xu}$ and Zhou [15], as just mentioned, and the other is proposed by Yang and Bi in [22], which has been applied to elliptic eigenvalue problems [22] and eigenvalue problem of electric field [23]. Compared with the first type, the second one is based on the shifted-inverse power method which makes itself more efficient, and starting from it, one can construct multiscale discretization scheme and parallel algorithm. So, this paper aims to establish a multi-scale discretization scheme based on the shifted-inverse power method, and an adaptive algorithm for the Steklov eigenvalue problem. The special work of this paper is as follows.

(1) It is well known that the Rayleigh quotient iteration method is a basic approach for solving matrix eigenvalue problems (see Algorithm 27.3 in [24]). The multi-scale discretization scheme established in this paper is a combination of the finite element method and the Rayleigh quotient iteration method. We prove that this scheme is efficient, and analyze elaborately that the constants appeared in the error estimates are independent of mesh size and the iterative time. It can be seen easily that a two-grid discretization scheme is a special case of the multi-scale algorithm by taking the iterative time equal to 2 (see Scheme 2 ). From the theoretical analysis (see Theorem 3.2) we know that when taking $H=\mathcal{O}(\sqrt[3]{h})$, the resulted solution can maintain an asymptotically optimal accuracy, while with the schemes in $[20,21]$, the resulted solution maintains an asymptotically optimal accuracy by taking $H=\mathcal{O}(\sqrt{h})$.

(2) The multi-scale discretization scheme is actually an iterative process. Since the approximate eigenvalue sequence obtained by the scheme converges to the exact eigenvalue (see Theorem 3.1, Corollary 3.4) and the constants appeared in its error estimates are independent of the mesh size and the iterative time, we can stop the iteration when the error between two neighboring approximate eigenvalues is less or equal to a given error tolerance. Thus, we establish an adaptive algorithm (see Scheme 3). With this adaptive scheme, first we solve an eigenvalue problem on a coarse grid, in each step after that we only need to solve a linear algebraic system on a fine grid. Compared with the existing adaptive method (e.g., see Algorithm 1 in [11]) which computes an eigenvalue problem in each step, our approach reduces the computational complexity.

The rest of this paper is organized as follows. In the subsequent section, some preliminaries needed in this paper are presented. In Section 3, a multi-scale and a two-grid discretization scheme are proposed, and the error estimates are also given. In Section 4, an adaptive algorithm is established. Finally, numerical experiments are provided to support our theoretical analysis.

\section{Preliminaries}

Let $H^{s}(\Omega)$ and $H^{s}(\partial \Omega)$ denote Sobolev spaces on $\Omega$ and $\partial \Omega$ with real order $s$, respectively. The norms in $H^{s}(\Omega)$ and $H^{s}(\partial \Omega)$ are denoted by $\|\cdot\|_{s}$ and $\|\cdot\|_{s, \partial \Omega}$, respectively.

Consider the model problem

$$
-\Delta u+u=0 \operatorname{in} \Omega, \quad \frac{\partial u}{\partial n}=\lambda u \text { on } \partial \Omega,
$$

where $\Omega \subset R^{2}$ is a polygonal domain and $\partial u / \partial n$ is the outward normal derivative on $\partial \Omega$. 
The weak form of (2.1) is given by the following: find $\lambda \in R, 0 \neq u \in H^{1}(\Omega)$, such that

$$
a(u, v)=\lambda b(u, v), \quad \forall v \in H^{1}(\Omega),
$$

where

$$
a(u, v)=\int_{\Omega} \nabla u \nabla v+u v d x, \quad b(u, v)=\int_{\partial \Omega} u v d s
$$

It is easy to know that $a(\cdot, \cdot)$ is a symmetric, continuous, and $H^{1}(\Omega)$-elliptic bilinear form on $H^{1}(\Omega) \times H^{1}(\Omega)$. So, we use $a(\cdot, \cdot)$ and $\|\cdot\|_{a}=\sqrt{a(\cdot, \cdot)}=\|\cdot\|_{1}$ as the inner product and norm on $H^{1}(\Omega)$, respectively. We use $b(\cdot, \cdot)$ and $\|\cdot\|_{b}=\sqrt{b(\cdot, \cdot)}$ as the inner product and norm on $L^{2}(\partial \Omega)$, respectively.

From [25], we know that (2.2) has a countable sequence of positive eigenvalues

$$
0<\lambda_{1} \leq \lambda_{2} \leq \lambda_{3} \leq \cdots \longrightarrow+\infty
$$

(here each eigenvalue occurs as many times as given by its multiplicity), and the corresponding eigenfunctions $u_{k} \in H^{1+r}(\Omega)(k=1,2, \ldots)$, where $r=1$ if $\Omega$ is convex, and $r<(\pi / \omega)$ which can be arbitrarily close to $\pi / \omega$ when $\Omega$ is concave (with $\omega$ being the largest inner angle of $\Omega, \omega<2 \pi)$.

Let $\pi_{h}$ be a regular triangulation of $\Omega$ with the mesh diameter $h$, and let $S^{h}$ be a piecewise polynomial space of degree $m(m \geq 1)$ defined on $\pi_{h}$.

The conforming finite element approximation of (2.2) is the following: find $\lambda_{h} \in R$, $0 \neq u_{h} \in S^{h} \subset H^{1}(\Omega)$, such that

$$
a\left(u_{h}, v\right)=\lambda_{h} b\left(u_{h}, v\right), \quad \forall v \in S^{h} .
$$

It is well known that (2.5) has a finite sequence of eigenvalues

$$
0<\lambda_{1, h} \leq \lambda_{2, h} \leq \cdots \lambda_{N_{h}, h}\left(N_{h}=\operatorname{dim} S^{h}\right),
$$

and their corresponding eigenfunctions are $u_{k, h}\left(k=1,2, \cdots, N_{h}\right)$.

Consider the following source problem (2.7) associated with (2.2) and the approximate source problem (2.8) associated with (2.5), respectively.

Find $u \in H^{1}(\Omega)$, such that

$$
a(u, v)=b(f, v), \quad \forall v \in H^{1}(\Omega) .
$$

Find $u_{h} \in S^{h}$, such that

$$
a\left(u_{h}, v\right)=b(f, v), \quad \forall v \in S^{h} .
$$

Several regular estimates of the Steklov eigenvalue problem are presented in the following lemma, which will be used in the sequel. 
Lemma 2.1. If $f \in L^{2}(\partial \Omega)$, then there exists a unique solution $u \in H^{1+(r / 2)}(\Omega)$ to (2.7) and

$$
\|u\|_{1+(r / 2)} \leq C_{p}\|f\|_{0, \partial \Omega}
$$

If $f \in H^{1 / 2}(\partial \Omega)$, then there exists a unique solution $u \in H^{1+r}(\Omega)$ to (2.7) and

$$
\|u\|_{1+r} \leq C_{p}\|f\|_{1 / 2, \partial \Omega} .
$$

If $f \in L^{2}(\partial \Omega)$, then there exists a unique solution $u \in H^{1}(\Omega)$ to (2.7) and

$$
\|u\|_{a} \leq C_{p}\|f\|_{0, \partial \Omega}
$$

where $C_{p}$ appeared in (2.9), (2.10), and (2.11) stands for a positive constant but may take different values.

Proof. As regards the proof of (2.9) and (2.10), see [26]. Since $a(\cdot, \cdot)$ is $H^{1}(\Omega)$-elliptic, from (2.7), it is easy to know that (2.11) is valid.

Thus, from (2.7), we can define the operator $A: L^{2}(\partial \Omega) \rightarrow H^{1}(\Omega)$ by

$$
a(A f, v)=b(f, v), \quad \forall v \in H^{1}(\Omega) .
$$

Similarly, from (2.8), we define the operator $A_{h}: L^{2}(\partial \Omega) \rightarrow S^{h} \subset H^{1}(\Omega)$ by

$$
a\left(A_{h} f, v\right)=b(f, v), \quad \forall v \in S^{h}
$$

It is obvious that $A: H^{1}(\Omega) \rightarrow H^{1}(\Omega)$ is a self-adjoint operator. In fact, for any $u, v \in H^{1}(\Omega)$, $a(A u, v)=b(u, v)=b(v, u)=a(A v, u)=a(u, A v)$. Analogously, $A_{h}$ is also a self-adjoint operator. Observe that $A f$ and $A_{h} f$ are the exact solution and the finite element solution of (2.7), respectively, and $a\left(A f-A_{h} f, v\right)=0, \forall v \in S^{h} \subset H^{1}(\Omega)$. Define the Ritz-Galerkin projection operator $P_{h}: H^{1}(\Omega) \rightarrow S^{h}$ by

$$
a\left(u-P_{h} u, v\right)=0, \quad \forall u \in H^{1}(\Omega), \forall v \in S^{h} .
$$

Thus, $\forall f \in H^{1}(\Omega)$,

$$
a\left(A_{h} f-P_{h}(A f), v\right)=a\left(A_{h} f-A f+A f-P_{h}(A f), v\right)=0, \quad \forall v \in S^{h}
$$


Therefore, $A_{h} f=P_{h} A f, \forall f \in H^{1}(\Omega)$, then $A_{h}=P_{h} A$. From Lemma 2.1 and the interpolation error estimate, we have

$$
\begin{aligned}
\left\|A_{h}-A\right\|_{a} & =\sup _{g \in H^{1}(\Omega)} \frac{\left\|\left(A_{h}-A\right) g\right\|_{a}}{\|g\|_{a}} \\
& =\sup _{g \in H^{1}(\Omega)} \frac{\left\|P_{h} A g-A g\right\|_{a}}{\|g\|_{a}} \\
& \leq \sup _{g \in H^{1}(\Omega)} \frac{C_{I} h^{r}\|A g\|_{1+r}}{\|g\|_{a}} \\
& \leq \sup _{g \in H^{1}(\Omega)} \frac{C_{I} h^{r}\|g\|_{a}}{\|g\|_{a}}=C_{I} h^{r} \rightarrow 0(h \rightarrow 0),
\end{aligned}
$$

where $C_{I}$ is the interpolation constant. It is clear that $A_{h}$ is a finite rank operator, then $A$ is a completely continuous operator. From [25,27], we know that (2.2) and (2.5) have the following equivalent operator forms, respectively:

$$
\begin{gathered}
A u=\mu u, \\
A_{h} u_{h}=\mu_{h} u_{h},
\end{gathered}
$$

where $\mu=1 / \lambda, \mu_{h}=1 / \lambda_{h}$. In this paper, $\mu_{k}$, and $\mu_{k, h}, \lambda_{k}$ and $\lambda_{k, h}$ are all called eigenvalues.

Suppose that the algebraic multiplicity of $\mu_{k}$ is equal to $q, \mu_{k}=\mu_{k+1}=\cdots=\mu_{k+q-1}$. Let $M\left(\mu_{k}\right)$ be the space spanned by all eigenfunctions corresponding to $\mu_{k}$ of $A$, and let $M_{h}\left(\mu_{k}\right)$ be the direct sum of eigenspaces corresponding to all eigenvalues of $A_{h}$ that converge to $\mu_{k}$. Let $\widehat{M}\left(\mu_{k}\right)=\left\{v: v \in M\left(\mu_{k}\right),\|v\|_{a}=1\right\}, \widehat{M}_{h}\left(\mu_{k}\right)=\left\{v: v \in M_{h}\left(\mu_{k}\right),\|v\|_{a}=1\right\}$. We also write $M\left(\lambda_{k}\right)=M\left(\mu_{k}\right), M_{h}\left(\lambda_{k}\right)=M_{h}\left(\mu_{k}\right), \widehat{M}\left(\lambda_{k}\right)=\widehat{M}\left(\mu_{k}\right)$, and $\widehat{M}_{h}\left(\lambda_{k}\right)=\widehat{M}_{h}\left(\mu_{k}\right)$.

Let

$$
\delta_{h}\left(\lambda_{k}\right)=\sup _{w \in \widehat{M}\left(\lambda_{k}\right)} \inf _{v \in S^{h}}\|w-v\|_{a}
$$

From Lemma 2.1, we know that $\widehat{M}\left(\lambda_{k}\right) \subset H^{1+r}(\Omega)$, then by the interpolation error estimate, we have

$$
\delta_{h}\left(\lambda_{k}\right) \leq C_{I} h^{r} \sup _{w \in \widehat{M}\left(\lambda_{k}\right)}\|w\|_{1+r}
$$

Lemma 2.2. Let $\lambda_{k, h}$ and $\lambda_{k}$ be the kth eigenvalue of (2.5) and (2.2), respectively. Then

$$
\lambda_{k} \leq \lambda_{k, h} \leq \lambda_{k}+C_{1} \delta_{h}^{2}\left(\lambda_{k}\right)
$$


for any eigenfunction $u_{k, h}$ corresponding to $\lambda_{k, h}$, satisfying $\left\|u_{k, h}\right\|_{a}=1$, there exists $u_{k} \in \widehat{M}\left(\lambda_{k}\right)$ such that

$$
\left\|u_{k, h}-u_{k}\right\|_{a} \leq C_{2} \delta_{h}\left(\lambda_{k}\right)
$$

and for any $u_{k} \in \widehat{M}\left(\lambda_{k}\right)$, there exists $u_{h} \in M_{h}\left(\lambda_{k}\right)$ such that

$$
\left\|u_{h}-u_{k}\right\|_{a} \leq C_{3} \delta_{h}\left(\lambda_{k}\right)
$$

where $C_{i}, i=1,2,3$ are constants independent of $h$.

Proof. By the argument in $[25,27]$, we can obtain the desired results.

The following lemma states a crucial property (but straightforward) of eigenvalue and eigenfunction approximation.

Lemma 2.3. Let $(\lambda, u)$ be an eigenpair of (2.2), then for any $w \in H^{1}(\Omega),\|w\|_{b} \neq 0$, the Rayleigh quotient $a(w, w) /\|w\|_{b}^{2}$ satisfies

$$
\frac{a(w, w)}{\|w\|_{b}^{2}}-\lambda=\frac{\|w-u\|_{a}^{2}}{\|w\|_{b}^{2}}-\lambda \frac{\|w-u\|_{b}^{2}}{\|w\|_{b}^{2}}
$$

Proof. See, for instance, Lemma 9.1 in [27] for details.

We also need the following basic estimate of shifted-inverse power method (see Theorem 3.2 in [22]).

Lemma 2.4. Let $\left(\mu_{0}, u_{0}\right)$ be an approximation for $\left(\mu_{k}, u_{k}\right)$, where $\mu_{0}$ is not an eigenvalue of $A_{h}$, and $u_{0} \in S^{h}$ with $\left\|u_{0}\right\|_{a}=1$. Suppose that $\max _{k \leq j \leq k+q-1}\left|\left(\mu_{j, h}-\mu_{k, h}\right) /\left(\mu_{0}-\mu_{j, h}\right)\right| \leq 1 / 2$, $\operatorname{dist}\left(u_{0}, M_{h}\left(\mu_{k}\right)\right) \leq 1 / 2,\left|\mu_{0}-\mu_{j, h}\right| \geq \rho / 2$ for $j \neq k, k+1, \ldots, k+q-1$, and $u \in S^{h}, u_{k}^{h} \in S^{h}$ satisfy

$$
\left(\mu_{0}-A_{h}\right) u=u_{0}, u_{k}^{h}=\frac{u}{\|u\|_{a}} .
$$

Then

$$
\operatorname{dist}\left(u_{k}^{h}, \widehat{M}_{h}\left(\mu_{k}\right)\right) \leq \frac{16}{\rho}\left|\mu_{0}-\mu_{k, h}\right| \operatorname{dist}\left(u_{0}, M_{h}\left(\mu_{k}\right)\right),
$$

where $\rho=\min _{\mu_{j} \neq \mu_{k}}\left|\mu_{j}-\mu_{k}\right|$ be the separation constant of the eigenvalue $\mu_{k}$.

For the multi-scale discretization scheme established in this paper, the conditions of Lemma 2.4 are satisfied which will be verified in the proof of Theorem 3.1. 


\section{Multi-Scale Discretization Scheme}

In this section, we combine the finite element method with the Rayleigh quotient iteration method and establish a multi-scale discretization scheme. Let $\left\{\pi_{h_{i}}\right\}_{1}^{l}$ be a family of regular meshes, $h_{i-1} \gg h_{i}$, and let $\left\{S^{h_{i}}\right\}_{1}^{l}$ be the conforming finite element spaces defined on $\left\{\pi_{h_{i}}\right\}_{1}^{l}$, and let $\pi_{H}=\pi_{h_{1}}, S^{H}=S^{h_{1}}, \pi_{h}=\pi_{h_{l}}$, and $S^{h}=S^{h_{l}}$.

Scheme 1 (multi-scale discretization scheme).

Step 1. Solve (2.2) on the $\pi_{H}$ : find $\lambda_{k, H} \in R, u_{k, H} \in S^{H}$ such that $\left\|u_{k, H}\right\|_{a}=1$ and

$$
a\left(u_{k, H}, v\right)=\lambda_{k, H} b\left(u_{k, H}, v\right), \quad \forall v \in S^{H} .
$$

Step 2. Execute the assignments: $u_{k}^{h_{1}} \Leftarrow u_{k, H}, \lambda_{k}^{h_{1}} \Leftarrow \lambda_{k, H}, i \Leftarrow 2$.

Step 3. Solve a linear system on the $\pi_{h_{i}}$ : find $\tilde{u} \in S^{h_{i}}$ such that

$$
a(\tilde{u}, v)-\lambda_{k}^{h_{i-1}} b(\tilde{u}, v)=b\left(u_{k}^{h_{i-1}}, v\right), \quad \forall v \in S^{h_{i}}
$$

And set $u_{k}^{h_{i}}=\tilde{u} /\left(\|\tilde{u}\|_{a}\right)$.

Step 4. Compute the Rayleigh quotient

$$
\lambda_{k}^{h_{i}}=\frac{a\left(u_{k}^{h_{i}}, u_{k}^{h_{i}}\right)}{b\left(u_{k}^{h_{i}}, u_{k}^{h_{i}}\right)} .
$$

Step 5. If $i=l$, then output $\left(\lambda_{k}^{h_{l}}, u_{k}^{h_{l}}\right)$, that is, $\left(\lambda_{k^{\prime}}^{h}, u_{k}^{h}\right)$, stop. Else, $i \Leftarrow i+1$, and return to Step 3 .

Theorem 3.1. Let $\left(\lambda_{k}^{h_{l}}, u_{k}^{h_{l}}\right)$ be obtained by Scheme 1. Assume that $\left(\lambda_{k}^{h_{l-1}}, u_{k}^{h_{l-1}}\right)$ approximate an eigenpair, $\left(\lambda_{k}, \bar{u}\right)$, of $(2.2), \bar{u} \in \widehat{M}\left(\lambda_{k}\right)$, and $\lambda_{k}^{h_{l-1}}-\lambda_{k}$ is a small quantity of lower order than $\lambda_{k, h_{l}}-\lambda_{k}$. Then there exists $u_{k} \in M\left(\lambda_{k}\right)$ such that

$$
\begin{gathered}
\left\|u_{k}^{h_{l}}-u_{k}\right\|_{a} \leq \frac{48}{\rho} C_{4} C_{5} \lambda_{k}\left|\lambda_{k}^{h_{l-1}}-\lambda_{k}\right| \operatorname{dist}\left(u_{k}^{h_{l-1}}, \widehat{M}\left(\lambda_{k}\right)\right)+3 C_{2} q \delta_{h_{l}}\left(\lambda_{k}\right), \\
\left|\lambda_{k}^{h_{l}}-\lambda_{k}\right| \leq 2 \lambda_{k}\left(1+C_{6} \lambda_{k}\right)\left\|u_{k}^{h_{l}}-u_{k}\right\|_{a^{\prime}}^{2} \quad l \geq 2
\end{gathered}
$$

where the constants $C_{4}, C_{5}$, and $C_{6}$ are stated in (3.6), (3.11), and (3.29), respectively.

Proof. We use Lemma 2.4 to complete the proof. First, we will verify that the conditions of Lemma 2.4 are satisfied.

Select $\mu_{0}=1 / \lambda_{k}^{h_{l-1}}$, and $u_{0}=\lambda_{k}^{h_{l-1}} A_{h_{l}} u_{k}^{h_{l-1}} /\left\|\lambda_{k}^{h_{l-1}} A_{h_{l}} u_{k}^{h_{l-1}}\right\|_{a}$. From (2.16), we know that $\left\|A_{h_{l}}-A\right\|_{a} \rightarrow 0\left(h_{l} \rightarrow 0\right)$, then there exists a constant $C_{4}$ independent of $h_{l}$ and $l$ such that

$$
\left\|A_{h_{l}} f\right\|_{a} \leq C_{4}\|f\|_{a^{\prime}} \quad \forall f \in H^{1}(\Omega) .
$$


Thus, by the assumption, we deduce that

$$
\begin{aligned}
\left\|\lambda_{k}^{h_{l-1}} A_{h_{l}} u_{k}^{h_{l-1}}-\bar{u}\right\|_{a} & \leq\left\|\lambda_{k}^{h_{l-1}} A_{h_{l}} u_{k}^{h_{l-1}}-\lambda_{k}^{h_{l-1}} A_{h_{l}} \bar{u}+\lambda_{k}^{h_{l-1}} A_{h_{l}} \bar{u}-\lambda_{k} A_{h_{l}} \bar{u}+\lambda_{k} A_{h_{l}} \bar{u}-\lambda_{k} A \bar{u}\right\|_{a} \\
& \leq C_{4} \lambda_{k}^{h_{l-1}}\left\|u_{k}^{h_{l-1}}-\bar{u}\right\|_{a}+C_{4}\left|\lambda_{k}^{h_{l-1}}-\lambda_{k}\right| \cdot\|\bar{u}\|_{a}+\lambda_{k}\left\|\left(A_{h_{l}}-A\right) \bar{u}\right\|_{a} .
\end{aligned}
$$

Note that in any normed space, for any nonzero $u, v \in S^{h}$, there holds

$$
\left\|\frac{u}{\|u\|}-\frac{v}{\|v\|}\right\| \leq 2 \frac{\|u-v\|}{\|u\|},\left\|\frac{u}{\|u\|}-\frac{v}{\|v\|}\right\| \leq 2 \frac{\|u-v\|}{\|v\|} .
$$

Hence, we have

$$
\begin{aligned}
\operatorname{dist}\left(u_{0}, \widehat{M}\left(\lambda_{k}\right)\right) & \leq\left\|u_{0}-\frac{\bar{u}}{\|\bar{u}\|_{a}}\right\|_{a} \leq 2\left\|\lambda_{k}^{h_{l-1}} A_{h_{l}} u_{k}^{h_{l-1}}-\bar{u}\right\|_{a} \\
& \leq 2\left\{C_{4} \lambda_{k}^{h_{l-1}}\left\|u_{k}^{h_{l-1}}-\bar{u}\right\|_{a}+C_{4}\left|\lambda_{k}^{h_{l-1}}-\lambda_{k}\right| \cdot\|\bar{u}\|_{a}+\lambda_{k}\left\|\left(A_{h_{l}}-A\right) \bar{u}\right\|_{a}\right\} .
\end{aligned}
$$

Using the triangle inequality and (2.22), we get

$$
\operatorname{dist}\left(u_{0}, M_{h_{l}}\left(\lambda_{k}\right)\right) \leq \operatorname{dist}\left(u_{0}, \widehat{M}\left(\lambda_{k}\right)\right)+C_{3} \delta_{h_{l}}\left(\lambda_{k}\right) .
$$

It follows from (2.20) that $\lambda_{k, h_{l}} \rightarrow \lambda_{k}\left(h_{l} \rightarrow 0\right)$, then by the assumption, we have

$$
\left|\mu_{0}-\mu_{k, h_{l}}\right|=\left|\frac{\lambda_{k}^{h_{l-1}}-\lambda_{k}+\lambda_{k}-\lambda_{k, h_{l}}}{\lambda_{k, h_{l}} \lambda_{k}^{h_{l-1}}}\right| \leq C_{5}\left|\lambda_{k}^{h_{l-1}}-\lambda_{k}\right|,
$$

where $C_{5}$ is a constant independent of $h_{l}$. By (2.13), we see that Step 3 in Scheme 1 is equivalent to the following:

$$
a(\tilde{u}, v)-\lambda_{k}^{h_{l-1}} a\left(A_{h_{l}} \tilde{u}, v\right)=a\left(A_{h_{l}} u_{k}^{h_{l-1}}, v\right), \quad \forall v \in S^{h},
$$

$u_{k}^{h_{l}}=\tilde{u} /\|\tilde{u}\|_{a}$, that is,

$$
\left(\frac{1}{\lambda_{k}^{h_{l-1}}}-A_{h_{l}}\right) \tilde{u}=\frac{1}{\lambda_{k}^{h_{l-1}}} A_{h_{l}} u_{k}^{h_{l-1}}, \quad u_{k}^{h_{l}}=\frac{\tilde{u}}{\|\tilde{u}\|_{a}} .
$$

Notice that $\left(1 / \lambda_{k}^{h_{l-1}}\right) A_{h_{l}} u_{k}^{h_{l-1}}=\left\|\left(1 / \lambda_{k}^{h_{l-1}}\right) A_{h_{l}} u_{k}^{h_{l-1}}\right\|_{a} u_{0}$ differs from $u_{0}$ by only a constant, then Step 3 is equivalent to

$$
\left(\frac{1}{\lambda_{k}^{h_{l-1}}}-A_{h_{l}}\right) \tilde{u}=u_{0}, \quad u_{k}^{h_{l}}=\frac{\tilde{u}}{\|\tilde{u}\|_{a}} .
$$


In fact, it is obvious that $u_{k}^{h_{l}}$ obtained by the above two formulae are the same. When $h_{l-1}$ is small enough, noting that $h_{l} \ll h_{l-1}$, from (3.10) and (3.9), we get

$$
\operatorname{dist}\left(u_{0}, M_{h_{l}}\left(\mu_{k}\right)\right) \leq \frac{1}{2}
$$

From (2.20), having in mind that $\lambda_{k}=\lambda_{k+1}=\cdots=\lambda_{k+q-1}$, we know that

$$
\lambda_{j, h_{l}}-\lambda_{k} \leq C_{1} \delta_{h_{l}}^{2}\left(\lambda_{k}\right) \quad(j=k, k+1, \ldots, k+q-1),
$$

then

$$
\left|\mu_{j, h_{l}}-\mu_{k, h_{l}}\right|=\left|\frac{\lambda_{k, h_{l}}-\lambda_{j, h_{l}}}{\lambda_{k, h_{l}} \lambda_{j, h_{l}}}\right|=\left|\frac{\lambda_{k, h_{l}}-\lambda_{k}+\lambda_{j}-\lambda_{j, h_{l}}}{\lambda_{k, h_{l}} \lambda_{j, h_{l}}}\right| .
$$

Combining (3.17) with (3.11) and noting that the quantity on the right-hand side of (3.17) is an infinitesimal of higher order comparing with $\lambda_{k}^{h_{l-1}}-\lambda_{k}$, we derive

$$
\max _{k \leq j \leq k+q-1}\left|\frac{\mu_{j, h_{l}}-\mu_{k, h_{l}}}{\mu_{0}-\mu_{j, h_{l}}}\right| \leq \frac{1}{2}
$$

Since $\rho$ is the separation constant, $h_{l-1}$ is small enough, and $h_{l} \ll h_{l-1}$, there holds

$$
\left|\mu_{0}-\mu_{j, h_{l}}\right| \geq \frac{\rho}{2}, \quad j \neq k, k+1, \ldots, k+q-1
$$

From the above arguments, we see that the conditions of Lemma 2.4 hold.

Next, we will prove that (3.4) and (3.5) are valid.

Substituting (3.10) and (3.11) into (2.25), we obtain

$$
\operatorname{dist}\left(u_{k}^{h_{l}}, \widehat{M}_{h_{l}}\left(\mu_{k}\right)\right) \leq \frac{16}{\rho} C_{5}\left|\lambda_{k}^{h_{l-1}}-\lambda_{k}\right|\left(\operatorname{dist}\left(u_{0}, \widehat{M}\left(\lambda_{k}\right)\right)+C_{3} \delta_{h_{l}}\left(\lambda_{k}\right)\right) .
$$

Let the eigenvectors $\left\{u_{j, h_{l}}\right\}_{k}^{k+q-1}$ be an orthonormal basis of $M_{h_{l}}\left(\lambda_{k}\right)$. Note that

$$
\operatorname{dist}\left(u_{k}^{h_{l}}, M_{h_{l}}\left(\lambda_{k}\right)\right)=\left\|u_{k}^{h_{l}}-\sum_{j=k}^{k+q-1} a\left(u_{k}^{h_{l}}, u_{j, h_{l}}\right) u_{j, h_{l}}\right\|_{a}
$$

Let

$$
u^{*}=\sum_{j=k}^{k+q-1} a\left(u_{k}^{h_{l}}, u_{j, h_{l}}\right) u_{j, h_{l}}
$$


it follows directly from (3.20) that

$$
\left\|u_{k}^{h_{l}}-u^{*}\right\|_{a} \leq \frac{16}{\rho} C_{5}\left|\lambda_{k}^{h_{l-1}}-\lambda_{k}\right|\left(\operatorname{dist}\left(u_{0}, \widehat{M}\left(\lambda_{k}\right)\right)+C_{3} \delta_{h_{l}}\left(\lambda_{k}\right)\right) .
$$

By Lemma 2.2, there exists $\left\{u_{j}^{0}\right\}_{k}^{k+q-1} \subset \widehat{M}\left(\lambda_{k}\right)$ making $u_{j, h_{l}}-u_{j}^{0}$ satisfy (2.21). Let

$$
u_{k}=\sum_{j=k}^{k+q-1} a\left(u_{k}^{h_{l}}, u_{j, h_{l}}\right) u_{j}^{0}
$$

then $u_{k} \in M\left(\lambda_{k}\right)$. Using (2.21), we deduce that

$$
\begin{aligned}
\left\|u^{*}-u_{k}\right\|_{a} & =\left\|\sum_{j=k}^{k+q-1} a\left(u_{k}^{h_{l}}, u_{j, h_{l}}\right)\left(u_{j, h_{l}}-u_{j}^{0}\right)\right\|_{a} \\
& \leq\left(\sum_{j=k}^{k+q-1}\left\|u_{j, h_{l}}-u_{j}^{0}\right\|_{a}^{2}\right)^{1 / 2} \\
& \leq C_{2} \sum_{j=k}^{k+q-1} \delta_{h_{l}}\left(\lambda_{j}\right) \leq C_{2} q \delta_{h_{l}}\left(\lambda_{k}\right) .
\end{aligned}
$$

Combining (3.23) with the above inequality, we have

$$
\begin{aligned}
\left\|u_{k}^{h_{l}}-u_{k}\right\|_{a} & \leq \frac{16}{\rho} C_{5}\left|\lambda_{k}^{h_{l-1}}-\lambda_{k}\right|\left(\operatorname{dist}\left(u_{0}, \widehat{M}\left(\lambda_{k}\right)\right)+C_{3} \delta_{h_{l}}\left(\lambda_{k}\right)\right)+C_{2} q \delta_{h_{l}}\left(\lambda_{k}\right) \\
& \leq \frac{16}{\rho} C_{5}\left|\lambda_{k}^{h_{l-1}}-\lambda_{k}\right| \operatorname{dist}\left(u_{0}, \widehat{M}\left(\lambda_{k}\right)\right)+2 C_{2} q \delta_{h_{l}}\left(\lambda_{k}\right) .
\end{aligned}
$$

It is obvious that there exists $u_{k}^{\prime} \in \widehat{M}\left(\lambda_{k}\right)$ such that

$$
\left\|u_{k}^{h_{l-1}}-u_{k}^{\prime}\right\|_{a}=\operatorname{dist}\left(u_{k}^{h_{l-1}}, \widehat{M}\left(\lambda_{k}\right)\right)
$$

$\lambda_{k} A u_{k}^{\prime}=u_{k}^{\prime} \in \widehat{M}\left(\lambda_{k}\right)$, and $\left\|\left(A_{h_{l}}-A\right) u_{k}^{\prime}\right\|_{a} \leq 1 / \lambda_{k} \delta_{h_{l}}\left(\lambda_{k}\right)$. 
Since $u_{0}=\lambda_{k}^{h_{l-1}} A_{h_{l}} u_{k}^{h_{l-1}} /\left\|\lambda_{k}^{h_{l-1}} A_{h_{l}} u_{k}^{h_{l-1}}\right\|_{a^{\prime}}$ from (2.23) and (3.8), we derive

$$
\begin{aligned}
\operatorname{dist}\left(u_{0}, \widehat{M}\left(\lambda_{k}\right)\right) \leq & 2 \operatorname{dist}\left(\lambda_{k}^{h_{l-1}} A_{h_{l}} u_{k}^{h_{l-1}}, \widehat{M}\left(\lambda_{k}\right)\right) \\
\leq & 2\left\|\lambda_{k}^{h_{l-1}} A_{h_{l}} u_{k}^{h_{l-1}}-\lambda_{k} A u_{k}^{\prime}\right\|_{a} \\
\leq & 2\left\|\left(\lambda_{k}^{h_{l-1}}-\lambda_{k}\right) A_{h_{l}} u_{k}^{h_{l-1}}\right\|_{a}+2\left\|\lambda_{k} A_{h_{l}}\left(u_{k}^{h_{l-1}}-u_{k}^{\prime}\right)\right\|_{a} \\
& +2\left\|\lambda_{k}\left(A_{h_{l}}-A\right) u_{k}^{\prime}\right\|_{a} \\
\leq & 2 C_{4}\left|\lambda_{k}^{h_{l-1}}-\lambda_{k}\right|+2 C_{4} \lambda_{k} \operatorname{dist}\left(u_{k}^{h_{l-1}}, \widehat{M}\left(\lambda_{k}\right)\right)+2 \delta_{h_{l}}\left(\lambda_{k}\right) \\
\leq & 3 C_{4} \lambda_{k} \operatorname{dist}\left(u_{k}^{h_{l-1}}, \widehat{M}\left(\lambda_{k}\right)\right)+2 \delta_{h_{l}}\left(\lambda_{k}\right) .
\end{aligned}
$$

Substituting (3.28) into (3.26), we obtain (3.4). Equation (3.4) indicates that $u_{k}^{h_{l}}$ converges to $u_{k}$ in the sense of norm $\|\cdot\|_{a}$, then from the trace theorem, we know that $u_{k}^{h_{l}}$ converges to $u_{k}$ in the sense of norm $\|\cdot\|_{b}$; thus, $1 /\left\|u_{k}^{h_{l}}\right\|_{b}^{2} \rightarrow 1 /\left\|u_{k}\right\|_{b}^{2}=\lambda_{k}$. Therefore, when $h_{l}$ is small enough, we have $1 /\left\|u_{k}^{h_{l}}\right\|_{b}^{2} \leq 2 \lambda_{k}$. Picking $w=u_{k}^{h_{l}}$ in (2.23), we get

$$
\begin{aligned}
\left|\lambda_{k}^{h_{l}}-\lambda_{k}\right| & \leq \frac{\left\|u_{k}^{h_{l}}-u_{k}\right\|_{a}^{2}}{\left\|u_{k}^{h_{l}}\right\|_{b}^{2}}+\lambda_{k} \frac{\left\|u_{k}^{h_{l}}-u_{k}\right\|_{b}^{2}}{\left\|u_{k}^{h_{l}}\right\|_{b}^{2}} \\
& \leq 2 \lambda_{k}\left\|u_{k}^{h_{l}}-u_{k}\right\|_{a}^{2}+2 \lambda_{k}^{2}\left\|u_{k}^{h_{l}}-u_{k}\right\|_{b}^{2} \\
& \leq 2 \lambda_{k}\left(1+C_{6} \lambda_{k}\right)\left\|u_{k}^{h_{l}}-u_{k}\right\|_{a^{\prime}}^{2}
\end{aligned}
$$

where the last inequality in the above holds due to the trace theorem, and the constant $C_{6}$ is independent of $h_{l}$.

Set $l=2$ and denote $H=h_{1}, h=h_{2}$, then we immediately get the following two-grid discretization scheme based on the shifted-inverse power method.

Scheme 2 (two-grid discretization scheme).

Step 1. Solve (2.2) on a coarse grid $\pi_{H}$ : find $\lambda_{k, H} \in R, u_{k, H} \in S^{H}$, such that $\left\|u_{k, H}\right\|_{a}=1$ and

$$
a\left(u_{k, H}, v\right)=\lambda_{k, H} b\left(u_{k, H}, v\right), \quad \forall v \in S^{H} .
$$

Step 2. Solve a linear system on a fine grid $\pi_{h}$ : find $\tilde{u} \in S^{h}$, such that

$$
a(\tilde{u}, v)-\lambda_{k, H} b(\widetilde{u}, v)=b\left(u_{k, H}, v\right), \quad \forall v \in S^{h}
$$

Set $u_{k}^{h}=\tilde{u} /\|\tilde{u}\|_{a}$. 
Step 3. Compute the Rayleigh quotient

$$
\lambda_{k}^{h}=\frac{a\left(u_{k}^{h}, u_{k}^{h}\right)}{b\left(u_{k}^{h}, u_{k}^{h}\right)} .
$$

Then from Theorem 3.1, we have the following error estimates for Scheme 2.

Theorem 3.2. Let $\left(\lambda_{k}^{h}, u_{k}^{h}\right)$ be obtained by Scheme 2. Then when $H$ is properly small and $h \ll H$, there exists $u_{k} \in M\left(\lambda_{k}\right)$ such that

$$
\begin{gathered}
\left\|u_{k}^{h}-u_{k}\right\|_{a} \leq \frac{48}{\rho} C_{4} C_{5} \lambda_{k}\left(\lambda_{k, H}-\lambda_{k}\right) \operatorname{dist}\left(u_{k, H}, \widehat{M}\left(\lambda_{k}\right)\right)+3 C_{2} q \delta_{h}\left(\lambda_{k}\right) \\
\left|\lambda_{k}^{h}-\lambda_{k}\right| \leq 2 \lambda_{k}\left(1+C_{6} \lambda_{k}\right)\left\|u_{k}^{h}-u_{k}\right\|_{a}^{2}
\end{gathered}
$$

Proof. Since $\left(\lambda_{k, H}, u_{k, H}\right)$ is obtained by Step 1 in Scheme 2, that is, $\left(\lambda_{k, H}, u_{k, H}\right)$ is a solution of (2.5) on the coarse grid $\pi_{H}$, it certainly approximates an eigenpair, $\left(\lambda_{k}, \bar{u}\right)$, of $(2.2), \bar{u} \in \widehat{M}\left(\lambda_{k}\right)$. Note that $\lambda_{k, h}$ is an eigenvalue of (2.5) on the fine grid $\pi_{h}$, so when $H$ is properly small and $h \ll H$, it is valid that $\lambda_{k, H}-\lambda_{k}$ is a small quantity of lower order than $\lambda_{k, h}-\lambda_{k}$. The above arguments imply that the conditions of Theorem 3.1 hold for $l=2$. Therefore, the desired results follow from Theorem 3.1.

Remark 3.3. It follows directly from (2.20) that

$$
\lambda_{k, H}-\lambda_{k} \leq C_{1} \delta_{H}^{2}\left(\lambda_{k}\right)
$$

And from (2.21), we know that $\operatorname{dist}\left(u_{k, H}, \widehat{M}\left(\lambda_{k}\right)\right) \leq C_{2} \delta_{H}\left(\lambda_{k}\right)$, then from Theorem 3.2, we get

$$
\left\|u_{k}^{h}-u_{k}\right\|_{a} \leq \frac{48}{\rho} C_{1} C_{2} C_{4} C_{5} \lambda_{k} \delta_{H}^{3}\left(\lambda_{k}\right)+3 C_{2} q \delta_{h}\left(\lambda_{k}\right)
$$

Substituting (2.19) into (3.36), we obtain

$$
\begin{aligned}
\left\|u_{k}^{h}-u_{k}\right\|_{a} \leq & \frac{48}{\rho} C_{2} C_{4} C_{5} \lambda_{k} C_{1} C_{I}^{3} H^{3 r}\left(\sup _{w \in \widehat{M}\left(\lambda_{k}\right)}\|w\|_{1+r}\right)^{3} \\
& +3 C_{2} q C_{I} h^{r} \sup _{w \in \widehat{M}\left(\lambda_{k}\right)}\|w\|_{1+r} .
\end{aligned}
$$

From (3.37) and (3.34), we can see that when we take $H=\mathcal{O}(\sqrt[3]{h})$, the resulted solution, $\left(\lambda_{k}^{h}, u_{k}^{h}\right)$, can maintain an asymptotically optimal accuracy. 
Corollary 3.4. Suppose that $h_{i}=h_{i-1}^{t_{i}}, t_{i} \in(1,3), i=2,3, \ldots, \sup _{i} t_{i}<3$. By using the triangle linear conforming element with regular meshes, when $h_{1}$, that is, $H$ is properly small, there exists $u_{k} \in M\left(\lambda_{k}\right)$ such that the following error estimates for Scheme 1 hold:

$$
\begin{gathered}
\left\|u_{k}^{h_{l}}-u_{k}\right\|_{a} \leq 6 q C_{2} C_{I} h_{l}^{r} \sup _{w \in \widehat{M}\left(\lambda_{k}\right)}\|w\|_{1+r} \\
\left|\lambda_{k}^{h_{l}}-\lambda_{k}\right| \leq 2 \lambda_{k}\left(1+C_{6} \lambda_{k}\right)\left\|u_{k}^{h_{l}}-u_{k}\right\|_{a^{\prime}}^{2} \quad l \geq 2 .
\end{gathered}
$$

Proof. We prove the results by using induction.

For $l=2$, we estimate $\lambda_{k, H}-\lambda_{k}$ and $\operatorname{dist}\left(u_{k, H}, \widehat{M}\left(\lambda_{k}\right)\right)$ in (3.33), respectively.

Combining (3.35) and (2.19) yields

$$
\lambda_{k, H}-\lambda_{k} \leq C_{1} C_{I}^{2} H^{2 r}\left(\sup _{w \in \widehat{M}\left(\lambda_{k}\right)}\|w\|_{1+r}\right)^{2} .
$$

From Lemma 2.2, and (3.8) we have

$$
\operatorname{dist}\left(u_{k, H}, \widehat{M}\left(\lambda_{k}\right)\right) \leq C_{2} \delta_{H}\left(\lambda_{k}\right) \leq C_{2} C_{I} H^{r} \sup _{w \in \widehat{M}\left(\lambda_{k}\right)}\|w\|_{1+r}
$$

Substituting the above estimates into (3.33), we get

$$
\begin{aligned}
\left\|u_{k}^{h}-u_{k}\right\|_{a} \leq & \frac{48}{\rho} C_{1} C_{2} C_{4} C_{5} \lambda_{k} C_{I}^{3} H^{3 r}\left(\sup _{w \in \widehat{M}\left(\lambda_{k}\right)}\|w\|_{1+r}\right)^{3} \\
& +3 q C_{2} C_{I} h^{r} \sup _{w \in \widehat{M}\left(\lambda_{k}\right)}\|w\|_{1+r} .
\end{aligned}
$$

By the assumption, $h=H^{t_{2}}, t_{2} \in(1,3)$, we can see that the first term on the right-hand side of (3.42) is a small quantity of higher order than the second term, hence,

$$
\left\|u_{k}^{h}-u_{k}\right\|_{a} \leq 6 q C_{2} C_{I} h^{r} \sup _{w \in \widehat{M}\left(\lambda_{k}\right)}\|w\|_{1+r}
$$

Suppose that (3.38), (3.39) hold for $l-1$, that is, there holds

$$
\begin{gathered}
\left\|u_{k}^{h_{l-1}}-u_{k}\right\|_{a} \leq 6 q C_{2} C_{I} h_{l-1}^{r} \sup _{w \in \widehat{M}\left(\lambda_{k}\right)}\|w\|_{1+r} \\
\left|\lambda_{k}^{h_{l-1}}-\lambda_{k}\right| \leq 2 \lambda_{k}\left(1+C_{6} \lambda_{k}\right)\left\|u_{k}^{h_{l-1}}-u_{k}\right\|_{a^{\prime}}^{2} \quad l \geq 2 .
\end{gathered}
$$

By (3.8), we get

$$
\operatorname{dist}\left(u_{k}^{h_{l-1}}, \widehat{M}\left(\lambda_{k}\right)\right) \leq 2\left\|u_{k}^{h_{l-1}}-u_{k}\right\|_{a} .
$$


Substituting (3.45) and (3.46) into (3.4), and noting (3.44), we deduce,

$$
\begin{aligned}
\left\|u_{k}^{h_{l}}-u_{k}\right\|_{a} & \leq \frac{192}{\rho} C_{4} C_{5} \lambda_{k}^{2}\left(1+C_{6} \lambda_{k}\right)\left(6 q C_{2} C_{I} \sup _{w \in \widehat{M}\left(\lambda_{k}\right)}\|w\|_{1+r}\right)^{3} h_{l-1}^{3 r}+3 C_{2} q \delta_{h_{l}}\left(\lambda_{k}\right) \\
& \leq \frac{192}{\rho} C_{4} C_{5} \lambda_{k}^{2}\left(1+C_{6} \lambda_{k}\right)\left(6 q C_{2} C_{I} \sup _{w \in \widehat{M}\left(\lambda_{k}\right)}\|w\|_{1+r}\right)^{3} h_{l-1}^{3 r}+3 q C_{2} C_{I} h_{l}^{r} \sup _{w \in \widehat{M}\left(\lambda_{k}\right)}\|w\|_{1+r} \\
& \leq 6 q C_{2} C_{I} h_{l}^{r} \sup _{w \in \widehat{M}\left(\lambda_{k}\right)}\|w\|_{1+r}
\end{aligned}
$$

that is, (3.38) holds. The inequality (3.39) follows directly from (3.29).

From Theorem 3.1 and Corollary 3.4, we can see that the error is independent of the mesh diameters and the iteration time, and Corollary 3.4 also shows that with Scheme 1, by using the linear element, the approximate eigenvalue sequence $\left\{\lambda_{k}^{h_{l}}\right\}$ converges to $\left\{\lambda_{k}\right\}$ as $l \rightarrow \infty$, and the convergence is quick. Hence, we have the reason to believe that $\left|\lambda_{k}^{h_{l}}-\lambda_{k}^{h_{l+1}}\right|<$ $\left|\lambda_{k}^{h_{l}}-\lambda_{k}^{h_{l-1}}\right|$. A simple calculation shows that

$$
\left|\lambda_{k}^{h_{l}}-\lambda_{k}\right| \leq\left|\lambda_{k}^{h_{l}}-\lambda_{k}^{h_{l+1}}\right|+\left|\lambda_{k}^{h_{l+1}}-\lambda_{k}\right| \leq\left|\lambda_{k}^{h_{l}}-\lambda_{k}^{h_{l-1}}\right|+\left|\lambda_{k}^{h_{l+1}}-\lambda_{k}\right|
$$

Note that in (3.48) $\left|\lambda_{k}^{h_{l+1}}-\lambda_{k}\right|$ is a small quantity of higher order. So we can use $\mid \lambda_{k}^{h_{l}}-$ $\lambda_{k}^{h_{l-1}} \mid<\varepsilon$ ( $\varepsilon$ is a given tolerate error) as a criteria to stop the iteration. Scheme 1 is actually an iterative process. It is natural to establish an adaptive algorithm based on Scheme 1.

Scheme 3 (adaptive algorithm). Give an error tolerance $\varepsilon$, an initial coarse grid $\pi_{H}$, and a grid $\pi_{h_{2}}$ derived from $\pi_{H}$.

Step 1. Solve (2.2) on the $\pi_{H}$ : find $\lambda_{k, H} \in R, u_{k, H} \in S^{H}$, such that $\left\|u_{k, H}\right\|_{a}=1$ and

$$
a\left(u_{k, H}, v\right)=\lambda_{k, H} b\left(u_{k, H}, v\right), \quad \forall v \in S^{H} .
$$

Step 2. Execute the assignments: $u_{k}^{h_{1}} \Leftarrow u_{k, H}, \lambda_{k}^{h_{1}} \Leftarrow \lambda_{k, H}, i \Leftarrow 2$.

Step 3. Solve a linear system on the $\pi_{h_{i}}$ : find $\tilde{u} \in S^{h_{i}}$, such that

$$
a(\tilde{u}, v)-\lambda_{k}^{h_{i-1}} b(\tilde{u}, v)=b\left(u_{k}^{h_{i-1}}, v\right), \quad \forall v \in S^{h_{i}}
$$

And set $u_{k}^{h_{i}}=\tilde{u} /\|\tilde{u}\|_{a}$. 
Table 1: Numerical eigenvalues on the square domain $[0,1] \times[0,1]$ by Scheme 2: set $h=\sqrt{2} / 512, h=$ $\mathcal{O}\left(H^{t}\right), t \in(1,3]$.

\begin{tabular}{lccccc}
\hline$H$ & $h$ & $\lambda_{1}^{h}$ & $\lambda_{2}^{h}$ & $\lambda_{3}^{h}$ & $\lambda_{4}^{h}$ \\
\hline$\sqrt{2} / 8$ & $\sqrt{2} / 512$ & 0.2400791223 & 1.4923055029 & 1.4923059934 & 2.0826626453 \\
$\sqrt{2} / 16$ & $\sqrt{2} / 512$ & 0.2400791223 & 1.4923054995 & 1.4923059872 & 2.0826625905 \\
$\sqrt{2} / 32$ & $\sqrt{2} / 512$ & 0.2400791223 & 1.4923054994 & 1.4923059871 & 2.0826625901 \\
\hline
\end{tabular}

Step 4. Compute the Rayleigh quotient

$$
\lambda_{k}^{h_{i}}=\frac{a\left(u_{k}^{h_{i}}, u_{k}^{h_{i}}\right)}{b\left(u_{k}^{h_{i}}, u_{k}^{h_{i}}\right)} .
$$

Step 5. If $\left|\lambda_{k}^{h_{i}}-\lambda_{k}^{h_{i-1}}\right|<\varepsilon$, then output $\left(\lambda_{k}^{h_{i}}, u_{k}^{h_{i}}\right)$, stop. Else, return to Step 6.

Step 6. Determine $t_{i+1}$ and $h_{i+1}=h_{i}^{t_{i+1}}$, then refine $\pi_{h_{i}}$ to get $\pi_{h_{i+1}}$. Set $i \Leftarrow i+1$, and return to Step 3.

\section{Numerical Experiments}

Example 4.1. We compute the first four approximate eigenvalues of (2.1) with the triangle linear finite element by using Scheme 2 on $\bar{\Omega}=[0,1] \times[0,1]$ and $[0,1] \times[0,1 / 2] \cup[0,1 / 2] \times$ $[1 / 2,1]$, respectively, [9] and [28] have proved that the non-conforming $E Q_{1}^{\text {rot }}$ element can provide the lower bound for the exact eigenvalues, and the minimum-maximum principle ensures that conforming finite elements can give the upper bound for the exact eigenvalues. So we compute the following ranges for the first four exact eigenvalues of (2.1) by the linear triangle element and the $E Q_{1}^{\text {rot }}$ element.

When $\bar{\Omega}=[0,1] \times[0,1]$ the first four exact eigenvalues

$$
\lambda_{1} \approx 0.24007909, \quad \lambda_{2} \approx 1.492303, \quad \lambda_{3} \approx 1.492303, \quad \lambda_{4} \approx 2.08265
$$

when $\bar{\Omega}=[0,1] \times[0,1 / 2] \cup[0,1 / 2] \times[1 / 2,1]$ the first four exact eigenvalues

$$
\lambda_{1} \approx 0.18296424, \quad \lambda_{2} \approx 0.8936, \quad \lambda_{3} \approx 1.68860, \quad \lambda_{4} \approx 3.2179 \text {. }
$$

When compute with Scheme 2, we adopt a uniform isosceles right triangulation along three directions to obtain the coarse grid $\pi_{H}$, and refine the coarse grid in a uniform way (each triangle is divided into four congruent subtriangles) repeatedly to obtain the fine grid $\pi_{h}$. Then we compute the approximate eigenvalues with MATLAB7.1. The numerical results are shown in Tables 1 and 2.

Remark 4.2. In Example 4.1, we set the diameter of fine grid $h=\sqrt{2} / 512$ and $H=\mathcal{O}(\sqrt[t]{h}), t \in$ $(1,3]$. From Table 1 we can see that the approximate eigenvalues obtain an asymptotically 
Table 2: Numerical eigenvalues on the L-shaped domain $[0,1] \times[0,1 / 2] \cup[0,1 / 2] \times[1 / 2,1]$ by Scheme 2 : set $h=\sqrt{2} / 512, h=\mathcal{O}\left(H^{t}\right), t \in(1,3]$.

\begin{tabular}{lccccc}
\hline$H$ & $h$ & $\lambda_{1}^{h}$ & $\lambda_{2}^{h}$ & $\lambda_{3}^{h}$ & $\lambda_{4}^{h}$ \\
\hline$\sqrt{2} / 8$ & $\sqrt{2} / 512$ & 0.1829642799 & 0.8937364938 & 1.6886068115 & 3.2179250611 \\
$\sqrt{2} / 16$ & $\sqrt{2} / 512$ & 0.1829642799 & 0.8937364004 & 1.6886067424 & 3.2179003466 \\
$\sqrt{2} / 32$ & $\sqrt{2} / 512$ & 0.1829642799 & 0.8937363984 & 1.6886067421 & 3.2179002027 \\
\hline
\end{tabular}

Table 3: Numerical eigenvalues on the square domain $\bar{\Omega}=[0,1] \times[0,1]$ by Scheme 3: set $h_{1}=H=1 / 8, h_{2}=$ $h_{1}^{2}, h_{i}=h_{i-1} / 2, i=3,4, \ldots$

\begin{tabular}{ccccc}
\hline & $\varepsilon=5 \times 10^{-7}$ & $\varepsilon=5 \times 10^{-5}$ & $\varepsilon=5 \times 10^{-5}$ & $\varepsilon=5 \times 10^{-5}$ \\
$l$ & $\lambda_{1}^{h_{l}}$ & $\lambda_{2}^{h_{l}}$ & $\lambda_{3}^{h_{l}}$ & $\lambda_{4}^{h_{l}}$ \\
\hline 1 & 0.2402262809 & 1.5014059516 & 1.5032096484 & 2.1452661239 \\
2 & 0.2400814379 & 1.4924542699 & 1.4924853962 & 2.0836406391 \\
3 & 0.2400796738 & 1.4923409581 & 1.4923487554 & 2.0828955752 \\
4 & 0.2400792326 & 1.4923125932 & 1.4923145438 & 2.0827091950 \\
5 & - & - & - & 2.0826625901 \\
6 & - & - & - & - \\
\hline
\end{tabular}

optimal accuracy. While with the two-grid discretization scheme in [20], to achieve the same accuracy the relationship between the diameter of coarse grid and that of fine grid should satisfy $H=\mathcal{O}(\sqrt[2]{h})$. For example, we carry out the computation on the same grids with $h=$ $\sqrt{2} / 512$ and $H=\sqrt{2} / 8$, by our scheme the resulted solution $\lambda_{1}^{h}=0.2400791223$ which has 7 significant digits, and by the scheme in [20] the resulted solution $\tilde{\lambda}_{1}^{h}=0.2400791543$ with 6 significant digits. Note that the computational complexities of these two methods are almost the same which indicates that our method is also efficient.

Remark 4.3. We program by using the finite element package of Chen [29] to solve Example 4.1. It costs 19.745 s by using Scheme 2, with $H=\sqrt{2} / 8$ and $h=\sqrt{2} / 512$, to get the first approximate eigenvalue $\lambda_{1}^{h}=0.2400791223$ of $(2.1)$ on the square $[0,1] \times[0,1]$. To illustrate the efficiency of our scheme we compute the first approximate eigenvalue on the fine grid with mesh diameter $h=\sqrt{2} / 512$ directly, and it costs 75.267s to get the same approximation. We also compare the computation times of our approach and direct calculation on the L-shaped domain $[0,1] \times[0,1 / 2] \cup[0,1 / 2] \times[1 / 2,1]$. By using Scheme 2 with $H=\sqrt{2} / 8$ and $h=\sqrt{2} / 512$, we spend 10.779 s to obtain $\lambda_{1}^{h}=0.1829642799$; while computing on the fine grid with mesh size $h=\sqrt{2} / 512$ directly it costs 31.986 s. From these comparisons we can see that our scheme is very efficient.

Example 4.4. We compute the first four approximate eigenvalues of (2.1) with the triangle linear finite element by using Scheme 3 on $[0,1] \times[0,1]$ and $[0,1] \times[0,1 / 2] \cup[0,1 / 2] \times[1 / 2,1]$, respectively. The results are listed in Tables 3 and 4, respectively. And in Tables 3 and 4, the symbol "-" means that we have obtained the approximate eigenvalues which meet the accuracy requirements and the iteration stops here.

Remark 4.5. In the research of numerical methods for Steklov eigenvalue problems, the boundary element method and the finite element method have been studied. However, we have not seen any literatures on the finite difference method, a major numerical method, for 
Table 4: Numerical eigenvalues on the L-shaped domain $\bar{\Omega}=[0,1] \times[0,1 / 2] \cup[0,1 / 2] \times[1 / 2,1]$ by Scheme 3 : set $h_{1}=H=1 / 8, h_{2}=h_{1}^{2}, h_{i}=h_{i-1} / 2, i=3,4, \ldots$.

\begin{tabular}{ccccc}
\hline & $\varepsilon=5 \times 10^{-7}$ & $\varepsilon=5 \times 10^{-4}$ & $\varepsilon=5 \times 10^{-5}$ & $\varepsilon=5 \times 10^{-4}$ \\
$l$ & $\lambda_{1}^{h_{l}}$ & $\lambda_{2}^{h_{l}}$ & $\lambda_{3}^{h_{l}}$ & $\lambda_{4}^{h_{l}}$ \\
\hline 1 & 0.1831328879 & 0.9211580591 & 1.7114443522 & 3.3645126465 \\
2 & 0.1829669801 & 0.8951626591 & 1.6889966003 & 3.2204599675 \\
3 & 0.1829649244 & 0.8942351070 & 1.6887001313 & 3.2185057817 \\
4 & 0.1829644089 & 0.8938763111 & 1.6886254813 & 3.2180214091 \\
5 & 0.1829642799 & - & 1.6886067421 & - \\
6 & - & - & - & - \\
\hline
\end{tabular}

Steklov eigenvalue problems. To apply the finite difference method to Steklov eigenvalue problems would be a new and interesting issue which is our next work.

\section{Concluding Remarks}

This paper discusses the Steklov eigenvalue problem, and establishes a new multi-scale discretization scheme and an adaptive algorithm. We prove that our approach is efficient. With the adaptive scheme, first we solve an eigenvalue problem on a coarse grid, in each step after that we only need to solve a linear algebraic system on a fine grid. Compared with the existing adaptive method which computes an eigenvalue problem in each step, our approach reduces the computational complexity. However, the a posteriori error indicator we used is a global estimate, thus, to establish a local a posteriori error estimate and improve our adaptive algorithm is our next goal.

\section{Acknowledgments}

The authors wish to thank $\mathrm{H}$. Li for his assistance with numerical experiments, and thank the editor and the referees for their valuable comments and suggestions that led to the improvement of this paper. This work is supported by the National Natural Science Foundation of China (Grant No. 11201093) and the Science and Technology Foundation of GuiZhou Province of China (LKS [2010] 01).

\section{References}

[1] S. Bergman and M. Schiffer, Kernel Functions and Elliptic Differential Equations in Mathematical Physics, Academic Press, New York, NY, USA, 1953.

[2] C. Conca, J. Planchard, and M. Vanninathan, Fluids and Periodic Structures, vol. 38 of RAM: Research in Applied Mathematics, John Wiley \& Sons, Chichester, UK, 1995.

[3] A. Bermúdez, R. Rodríguez, and D. Santamarina, "A finite element solution of an added mass formulation for coupled fluid-solid vibrations," Numerische Mathematik, vol. 87, no. 2, pp. 201-227, 2000.

[4] J. Huang and T. Lü, "The mechanical quadrature methods and their extrapolation for solving BIE of Steklov eigenvalue problems," Journal of Computational Mathematics, vol. 22, no. 5, pp. 719-726, 2004.

[5] A. B. Andreev and T. D. Todorov, "Isoparametric finite-element approximation of a Steklov eigenvalue problem," IMA Journal of Numerical Analysis, vol. 24, no. 2, pp. 309-322, 2004.

[6] M. G. Armentano and C. Padra, "A posteriori error estimates for the Steklov eigenvalue problem," Applied Numerical Mathematics, vol. 58, no. 5, pp. 593-601, 2008. 
[7] M. Li, Q. Lin, and S. Zhang, "Extrapolation and superconvergence of the Steklov eigenvalue problem," Advances in Computational Mathematics, vol. 33, no. 1, pp. 25-44, 2010.

[8] A. Alonso and A. Dello Russo, "Spectral approximation of variationally-posed eigenvalue problems by nonconforming methods," Journal of Computational and Applied Mathematics, vol. 223, no. 1, pp. 177-197, 2009.

[9] Y. Yang, Q. Li, and S. Li, “Nonconforming finite element approximations of the Steklov eigenvalue problem," Applied Numerical Mathematics, vol. 59, no. 10, pp. 2388-2401, 2009.

[10] A. Dello Russo and A. E. Alonso, "A posteriori error estimates for nonconforming approximations of Steklov eigenvalue problems," Computers \& Mathematics with Applications, vol. 62, no. 11, pp. 41004117, 2011.

[11] E. M. Garau and P. Morin, "Convergence and quasi-optimality of adaptive FEM for Steklov eigenvalue problems," IMA Journal of Numerical Analysis, vol. 31, no. 3, pp. 914-946, 2011.

[12] H. Bi, S. Ren, and Y. Yang, "Conforming finite element approximations for a fourth-order Steklov eigenvalue problem," Mathematical Problems in Engineering, vol. 2011, Article ID 873152, 13 pages, 2011.

[13] J. Xu, "A new class of iterative methods for nonselfadjoint or indefinite problems," SIAM Journal on Numerical Analysis, vol. 29, no. 2, pp. 303-319, 1992.

[14] J. Xu, "Two-grid discretization techniques for linear and nonlinear PDEs," SIAM Journal on Numerical Analysis, vol. 33, no. 5, pp. 1759-1777, 1996.

[15] J. Xu and A. Zhou, "A two-grid discretization scheme for eigenvalue problems," Mathematics of Computation, vol. 70, no. 233, pp. 17-25, 2001.

[16] C. S. Chien and B. W. Jeng, "A two-grid finite element discretizatin scheme for nonlinear eigenvalue problems," Computational Methods, pp. 1951-1955.

[17] H. Chen, F. Liu, and A. Zhou, "A two-scale higher-order finite element discretization for Schrödinger equation," Journal of Computational Mathematics, vol. 27, no. 2-3, pp. 315-337, 2009.

[18] X. G. Gong, L. Shen, D. Zhang, and A. Zhou, "Finite element approximations for Schrödinger equations with applications to electronic structure computations," Journal of Computational Mathematics, vol. 26, no. 3, pp. 310-323, 2008.

[19] T. Utnes, "Two-grid finite element formulations of the incompressible Navier-Stokes equations," Communications in Numerical Methods in Engineering, vol. 13, no. 8, pp. 675-684, 1997.

[20] Q. Li and Y. Yang, "A two-grid discretization scheme for the Steklov eigenvalue problem," Journal of Applied Mathematics and Computing, vol. 36, no. 1-2, pp. 129-139, 2011.

[21] H. Bi and Y. Yang, "A two-grid method of the non-conforming Crouzeix-Raviart element for the Steklov eigenvalue problem," Applied Mathematics and Computation, vol. 217, no. 23, pp. 9669-9678, 2011.

[22] Y. Yang and H. Bi, "Two-grid finite element discretization schemes based on shifted-inverse power method for elliptic eigenvalue problems," SIAM Journal on Numerical Analysis, vol. 49, no. 4, pp. 1602 1624, 2011.

[23] Y. Yang, W. Jiang, Y. Zhang, W. Wang, and H. Bi, "A two-scale discretization scheme for mixed variational formulation of eigenvalue problem," Abstract and Applied Analysis, vol. 2012, Article ID 812914, 29 pages, 2012.

[24] L. N. Trefethen and D. Bau, III, Numerical Linear Algebra, Society for Industrial and Applied Mathematics (SIAM), Philadelphia, Pa, USA, 1997.

[25] J. H. Bramble and J. E. Osborn, "Approximation of Steklov eigenvalues of non-selfadjoint second order elliptic operators," in The Mathematical Foundations of the Finite Element Method with Applications to Partial Differential Equations, pp. 387-408, Academic Press, New York, NY, USA, 1972.

[26] M. Dauge, Elliptic Boundary Value Problems on Corner Domains, vol. 1341 of Lecture Notes in Mathematics, Springer, Berlin, Germany, 1988.

[27] I. Babuška and J. Osborn, "Eigenvalue problems," in Finite Element Methods (Part 1), P. G. Ciarlet and J. L. Lions, Eds., vol. 2 of Handbook of Numerical Analysis II, pp. 641-787, North-Holland, Amsterdam, The Netherlands, 1991.

[28] Q. Lin, L. Tobiska, and A. Zhou, "Superconvergence and extrapolation of non-conforming low order finite elements applied to the Poisson equation," IMA Journal of Numerical Analysis, vol. 25, no. 1, pp. 160-181, 2005.

[29] L. Chen, IFEM: An Innovative Finite Element Methods Package in MATLAB, 2008. 


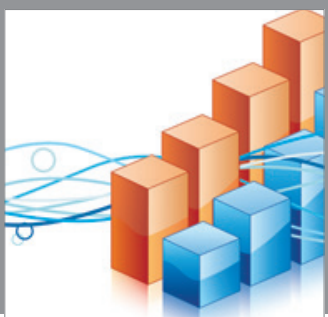

Advances in

Operations Research

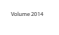

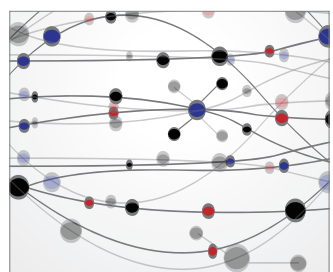

\section{The Scientific} World Journal
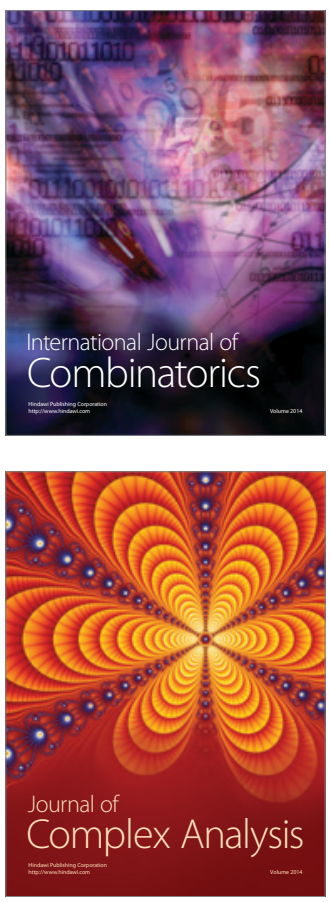

International Journal of

Mathematics and

Mathematical

Sciences
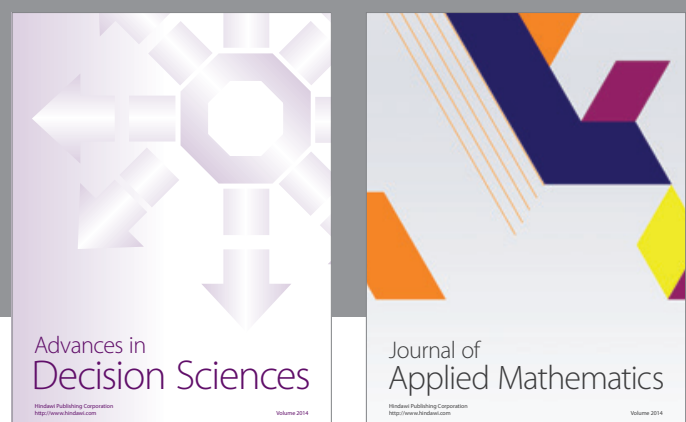

Journal of

Applied Mathematics
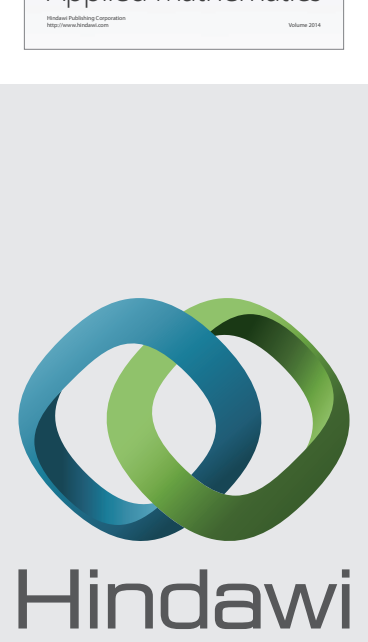

Submit your manuscripts at http://www.hindawi.com
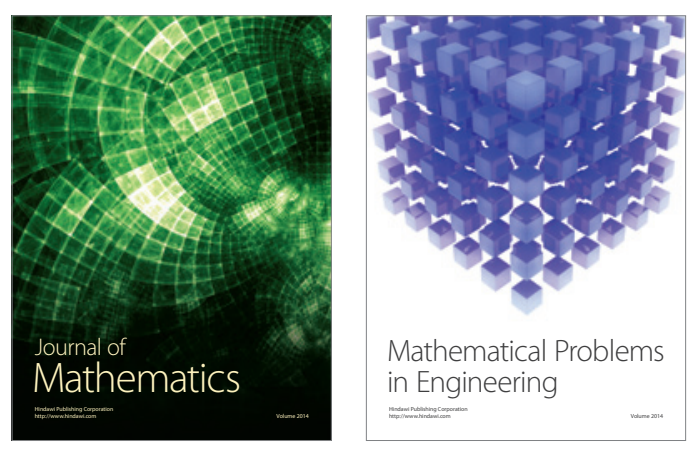

Mathematical Problems in Engineering
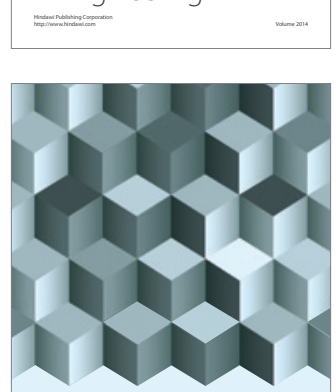

Journal of

Function Spaces
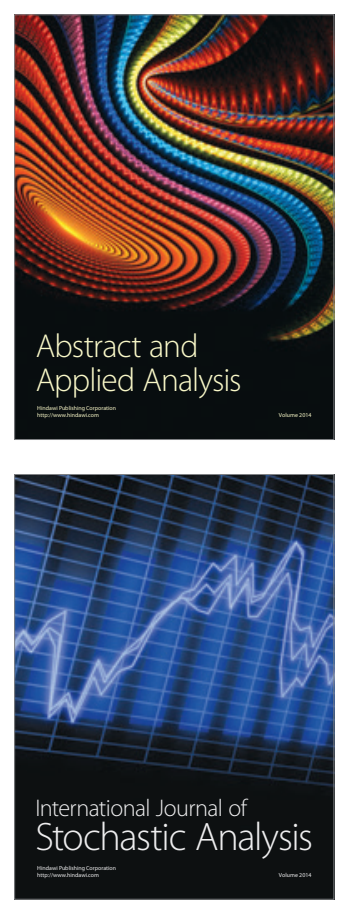

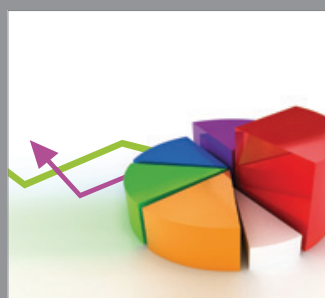

ournal of

Probability and Statistics

Promensencen
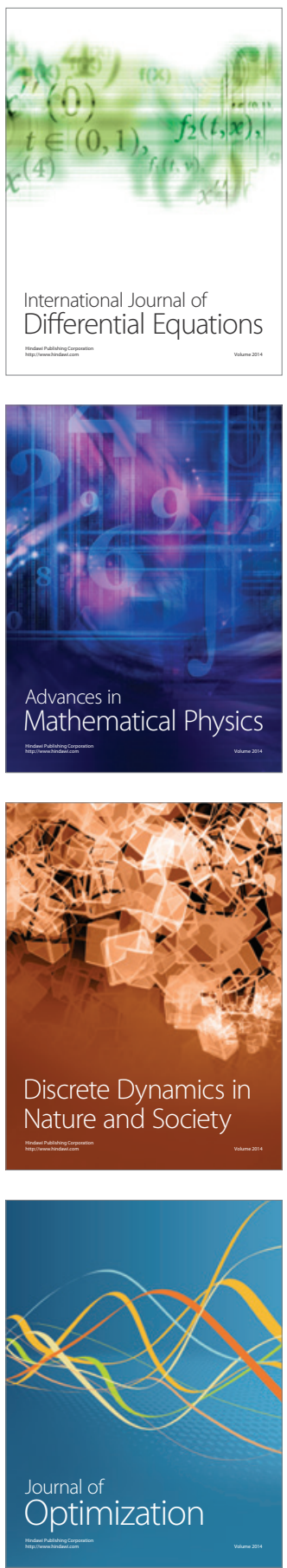